027.

Into the Fray: Integration of Emergency Medical Services and Special Weapons and Tactics (SWAT) Teams

Kevin Reese, $M D$, * Jeffrey Jones, $M D$, Greg Kenepp, Jon Krohmer, $M D$ Emergency Medicine Residency Program, Butterworth Hospital, Grand Rapids, Michigan USA

Objective: To determine current experience and training of emergency medical support personnel for special weapons and tactics (SWAT) teams in North America.

Methods: This is a cross-sectional, epidemiologic survey sent to SWAT unit commanders from the 200 largest metropolitan areas. Questions included basic demographics, specialized training of EMS personnel, and where they are deployed during tactical operations. Unit commanders also were asked to estimate the number and type of injuries sustained during tactical operations and to list any recommendations they have to improve the EMS response.

Results: A total of 150 surveys $(75 \%)$ were completed; representing 190 SWAT teams. The mean number of personnel on a tactical team was 24 (range 7-85); including two medical support personnel (range 0-12). Medical personnel were usually civilian paramedics or EMTs $(53 \%)$, followed by police officerEMTs $(27 \%)$, and tactical-EMTs $(17 \%)$. Thirty-eight percent of respondents reported a significant injury occurring during their tactical operations over the past 24 months. Common injuries included gunshot wounds, chemical exposure, and fractures. However, $78 \%(117 / 150)$ of respondents did not have a medical director, $47 \%$ of medical personnel had no specialized field training (e.g., triage, evacuation), and $23 \%$ of teams did not have an EMS preplan or protocol.

Conclusion: This is the first report to describe the availability and training of emergency-care providers for tactical law enforcement in North America. The results suggest a need for established EMS protocols and specialized tactical medical training, especially in large metropolitan areas.
063.

\section{Neighborhood-Based Variations in Treatment Provided by an Urban EMS System}

Theodore R. Delbridge, $M D, M P H$, * Thomas E. Auble, $P h D$,

Donald M. Yealy, $M D$, Herbert G. Garrison, $M D, M P H$

University of Pittsburgh, Division of Emergency Medicine, Pittsburgh, Pennsylvania USA

Objective: We sought to identify and characterize inter-neighborhood variation in the level of treatment provided within an urban EMS system for specific patient complaints.

Methods: This was a retrospective study of patient records from an urban, municipal, advanced life support EMS system for a six-month period. We extracted the incident identification number, location code, incident type, patient age and gender, treatment provided, and transport disposition from each record and entered them into a database. The city was divided into 19 geographically based neighborhoods, and socioeconomic data for each were determined from U.S. census reports. We utilized $c^{2}$ for trends analyses $(\alpha=0.05)$ to detect differences in transport rates and levels of treatment for patients from each neighborhood.

Results: We reviewed the records for all 23,100 EMS patient contacts during the study period. Of these, $18.9 \%$ of patients were treated and not transported to a hospital, $10.2 \%$ were transported without treatment, $48.2 \%$ received BLS-level treatment during transport, and $22.6 \%$ received ALS-level treatment during transport. For each neighborhood, the percentage of patients transported with ALS treatment ranged from $12 \%$ to $41 \%$. Nonspecific medical illness, chest pain, shortness of breath, and abdominal pain were the four most common patient complaints. For these complaints, neighborhood median income and population $>69$ years old were associated with a greater likelihood of ALS treatment during transport ( $p=0.001$ each). However, except for shortness-of-breath cases, neighborhood percent minority population was associated with a decreased likelihood of receiving ALS treatment during transport $(p<0.001)$. We did not find an association between any of the socioeconomic variables examined and rates of nontransport.

Conclusions: The results indicate that, despite similar complaints, the level of treatment provided varies among neighborhoods within a given EMS system. The factors contributing to this variation require identification. 